\title{
A COMPLETE EXTREMAL DISTANCE PROBLEM ON OPEN RIEMANN SURFACES ${ }^{1}$
}

\author{
BY A. MARDEN AND B. RODIN
}

Communicated by L. Bers, November 10, 1965

Partition the boundary contours of a compact bordered Riemann surface $\bar{W}$ into four disjoint sets $\alpha_{0}, \alpha, \beta, \gamma$ with $\alpha_{0}$ and $\alpha$ nonempty. Let $F$ consist of all arcs in $\bar{W}-\gamma$ which have initial point in $\alpha_{0}$ and endpoint in $\alpha$. Let $F^{*}$ consist of all cycles in $\bar{W}-\beta$ which separate $\alpha_{0}$ from $\alpha$. Determine the harmonic function $u$ in $\bar{W}$ by the boundary conditions $u=0$ on $\alpha_{0}, u=1$ on $\alpha, \partial u / \partial n=0$ along $\gamma$, and $u$ is constant on each contour $\beta_{i}$ in $\beta$ with the constant so chosen that $\int_{\beta_{i}} d u^{*}=0$. Then $\lambda(F)=\lambda^{-1}\left(F^{*}\right)=\|d u\|^{-2}$ where $\lambda(\cdot)$ denotes the extremal length and $\|\cdot\|^{2}$ denotes the Dirichlet norm. This result is implicit in the fundamental work of Ahlfors-Beurling [1]. Observe that if $\bar{W}$ is planar and $\alpha_{0}, \alpha$ are single contours then $\exp 2 \pi\left(u+i u^{*}\right) /\|d u\|^{2}$ is a conformal map of Int $\bar{W}$ into $1<|z|<\exp 2 \pi /\|d u\|^{2}$, the components of $\beta$ going onto circular slits and the components in $\gamma$ onto radial slits.

The purpose of this note is to announce a complete generalization of the above result which is valid for arbitrary open Riemann surfaces. As a consequence of our work we obtain a new class of conformal mappings of plane regions onto "extremal" slit annuli analogous to the situation described above. These results and their proofs will be published in a forthcoming paper [2].

We begin with an open Riemann surface $W$ and partition its Kerékjártó-Stoilöw ideal boundary into four disjoint sets $\alpha_{\theta}, \alpha, \beta, \gamma$ with $\alpha_{0}$ and $\alpha$ nonempty. For technical reasons we assume that $\alpha_{0}, \alpha, \alpha_{0} \cup \alpha \cup \beta$ are closed subsets of the Kerékjártó-Stoilöw compactification $\hat{W}$ of $W$. Let $\mathcal{F}$ be the family of arcs in $\hat{W}-\gamma$ with initial points in $\alpha_{0}$ and end points in $\alpha$. Let $\mathcal{F}^{*}$ consist of all suitably orientated $\tau$ such that $\tau$ is a countable union of closed curves in $\hat{W}-\alpha_{0}$ $-\alpha-\beta$, all limit points of $\tau$ are contained in $\gamma$, and no component of $\hat{W}-\gamma-\tau$ contains points in both $\alpha_{0}$ and $\alpha$. There is a natural definition for $\lambda(\mathscr{F}), \lambda\left(F^{*}\right)$ obtained by replacing each curve $\tau \subset \hat{W}$ by the curve $\tau \cap W$. An $H D$-function $u$ on $W$ is constructed which generalizes the $u$ defined above for compact bordered surfaces. The actual definition of $u$ uses a noncompact exhaustion of $W$ "in the direction

1 This work was supported in part by the National Science Foundation under grant GP 2280 at the University of Minnesota and GP 4106 at the University of California, San Diego. 
of $\gamma$. " We shall omit the precise definition here in favor of characterizing $u$ by an extremal property (see below). Our main result is the

Theorem. (a) $\lambda(\Im)=\|d u\|^{-2}$ and (b) $\lambda\left(F^{*}\right)=\|d u\|^{2}$.

For the proof of (a) one obtains $\lambda(F) \geqq\|d u\|^{-2}$ fairly easily. The opposite inequality depends on a highly topological continuity method for extremal length in which arcs in an exhaustion of $W$ are pieced together to form an arc in $\hat{W}$. This method is ascribed to Beurling and was developed by Strebel [6]. Using it, Strebel proved (a) in the case $\beta=\phi$. Even in this special case part (b) is new; it asserts that the conjugate extremal distance between two ends of an open Riemann surface is the extremal length of the class of curves which separate these ends. Part (b) is proven by establishing a generalized Green's formula which implies that $\int_{c} d u^{*}=\|d u\|^{2}$ for almost all curves $c \in F^{*}$. ("Almost all" means with the exception of a family of curves of infinite extremal length.)

As immediate corollaries, several uniqueness properties of $u$ are obtained. For example, $u$ minimizes $\|d h\|$ among all harmonic $h$ which satisfy $\int_{c} d h \geqq 1$ for almost all $c \in \mathcal{F}$.

Let the ideal point $\alpha_{0}$ be replaced by a point in $W$. A harmonic function $p$ can be constructed with the boundary behavior of $u$ near $\alpha, \beta, \gamma$ and with a logarithmic singularity at $\alpha_{0}$ of period $2 \pi$. $p$ generalizes the capacity function of Sario. We show that even on a nonplanar surface there always exists a boundary component of maximal generalized capacity. The functions $\exp 2 \pi\left(u+i u^{*}\right) /\|d u\|^{2}$ and $\exp \left(p+i p^{*}\right)$ give conformal mappings when $W$ is planar and $\alpha_{0}, \alpha$ are single components. Their images will be called extremal slit annuli or disks respectively. We show that (i) the area of the slits is 0 , (ii) the image of a boundary component in $\gamma$ is a radial slit or a point, (iii) the image of a component in $\beta$ which is isolated from $\gamma$ is a circular slit or a point, and (iv) in many other cases the image is circular with radial incisions.

Our results imply the now classical properties of extremal circular slit annuli $(\gamma=\phi)$ as found in Reich-Warschawski [4], [5], and of extremal radial slit annuli $(\beta=\phi)$ obtained by Strebel [6], [7] and Reich [3]. Even in these classical cases, however, the following corollary of the theorem is new.

Suppose $A$ is a plane region contained in $1<|z|<R$. Set $\rho(z)$ $=(|z| \log R)^{-1}$ and $k=2 \pi / \log R$. The following are equivalent :

(1) $A$ is an extremal slit annulus of radii $1, R$ (i.e. the function $u$ constructed for $A$ is $(\log |z|) / \log R)$.

(2) $\int_{\sigma} \rho|d z| \geqq 1$ and $\int_{\tau} \rho|d z| \geqq k$ for almost all $\sigma \in \mathcal{F}, \tau \in \mathcal{F}^{*}$. 
(3) $\lambda(F) \leqq k^{-1}$ and $\int_{\sigma} \rho|d z| \geqq 1$ for almost all $\sigma \in \mathcal{F}$.

(4) $\lambda(F) \geqq k^{-1}$ and $\int_{r} \rho|d z| \geqq k$ for almost all $\tau \in F^{*}$.

If $\beta=\phi$ or $\gamma=\phi$ then (3), (4) can be replaced by the single condition $\lambda(\mathcal{F})=k^{-1}$.

\section{REFERENCES}

1. L. Ahlfors and A. Beurling, Conformal invariants and function-theoretic null sets, Acta Math. 83 (1950), 101-129.

2. A. Marden and B. Rodin, Extremal and conjugate extremal distance on open Riemann surfaces with applications to circular-radial slit mappings, Acta Math. (to appear).

3. E. Reich, On radial slit mappings, Ann. Acad. Sci. Fenn. Ser. A 296 (1961), 1-12.

4. E. Reich and S. Warschawski, On canonical conformal maps on regions of arbitrary connectivity, Pacific J. Math. 10 (1960), 965-985.

5. - Canonical conformal maps onto a circular slit annulus, Scripta Math. 25 (1960), 137-146.

6. K. Strebel, Die extremale Distanz zweier Enden einer Riemannschen Fläche, Ann. Acad. Sci. Fenn. Ser. A 179 (1955), 1-21.

7. - A remark on the extremal distance of two boundary components, Proc. Nat. Acad. Sci. U.S.A. 40 (1954), 842-844.

UNIVERSITY OF MINNESOTA AND

University of California, San Diego 